\title{
Two cases of Kawasaki disease presented with acute febrile jaundice
}

\author{
Ayşe Kaman¹, Türkan Aydın-Teke ${ }^{1}$, Zeynep Gökçe Gayretli-Aydın¹, Fatma Nur Öz¹, Özge \\ Metin-Akcan ${ }^{1}$, Deniz Eriș ${ }^{2}$, Gönül Tanır ${ }^{1}$ \\ ${ }^{1}$ Division of Pediatric Infectious Diseases, ${ }^{2}$ Department of Pediatrics, Dr. Sami Ulus Maternity and Children's Training \\ and Research Hospital, Ankara, Turkey.E-mail: ayse092003@yahoo.com \\ Received: 18 th January 2016, Accepted: 28th January 2016
}

\begin{abstract}
SUMMARY: Kaman A, Aydın-Teke T, Gayretli-Aydın ZG, Öz FN, Metin-Akcan Ö, Eriş D, Tanır G. Two cases of Kawasaki disease presented with acute febrile jaundice. Turk J Pediatr 2017; 59: 84-86.

Kawasaki disease is an acute, systemic vasculitis of unknown etiology. Although gastrointestinal involvement does not belong to the classic diagnostic criteria; diarrhea, abdominal pain, hepatic dysfunction, hydrops of gallbladder, and acute febrile cholestatic jaundice are reported in patients with Kawasaki disease. We describe here two cases presented with fever, and acute jaundice as initial features of Kawasaki disease.
\end{abstract}

Key words: children, cholestasis, jaundice, Kawasaki disease.

Kawasaki disease (KD) is an acute, systemic vasculitis of unknown etiology that generally affects children younger than five years of age. The diagnosis of KD is based on the clinical criteria that include the most frequent and characteristic features of the disease. The presentation with atypical or incomplete KD findings, is a leading cause of delayed diagnosis. Gastrointestinal tract involvement is defined among the noncardiac findings of disease. ${ }^{1}$ In this report we described two patients with an unusual onset of $\mathrm{KD}$, characterized by fever, and acute jaundice.

\section{Case Reports}

\section{Case 1}

A six-year-old boy was admitted to hospital with complaints of fever for seven days, abdominal pain and jaundice. Physical examination revealed a fever of $38.5^{\circ} \mathrm{C}$, irritability, jaundice and abdominal tenderness. The medical history of the patient was unremarkable. Laboratory findings were as follows: hemoglobin concentration $9.1 \mathrm{~g} / \mathrm{dl}$; white blood cell count (WBC) $8700 / \mathrm{mm}^{3}$; platelet count $182.000 /$ $\mathrm{mm}^{3}$; erythrocyte sedimentation rate (ESR), $55 \mathrm{~mm} / \mathrm{h}(0-10 \mathrm{~mm} / \mathrm{h})$ and C-reactive protein (CRP), $160 \mathrm{mg} / \mathrm{dl}(0-8 \mathrm{mg} / \mathrm{dl})$, serum alanin aminotransferase (ALT) $42 \mathrm{U} / \mathrm{L}(5-40 \mathrm{U} / \mathrm{L})$, aspartate aminotransferase (AST) $29 \mathrm{U} / \mathrm{L}$
(8-33 U/L), total bilirubin $4.8 \mathrm{mg} / \mathrm{dl}(0-2$ $\mathrm{mg} / \mathrm{dl})$, direct bilirubin $4 \mathrm{mg} / \mathrm{dl}(0-0.5 \mathrm{mg} /$ dl), gamma glutamyl transferase (GGT) 168 $\mathrm{U} / \mathrm{L}(<23 \mathrm{U} / \mathrm{L})$, albumin $2.8 \mathrm{~g} / \mathrm{dl}$ (3.1-4.8 $\mathrm{g} / \mathrm{dl})$. Urinalysis revealed the presence of pyuria. Serological tests for hepatitis A, B, C, Epstein-Barr virus (EBV) and cytomegalovirus were negative for acute infections. Abdominal ultrasonography showed that subhepatic and pelvic fluid, normal gallbladder, bile ducts and pancreas. Minimal bilateral pleural effusion was detected on chest ultrasonography. Ceftriaxone treatment was commenced for possible enteric fever or urinary tract infection. Cultures of blood, urine and throat had resulted as negative thereafter but fever of the patient persisted despite broad-spectrum antibiotic treatment. Echocardiography was performed in suspicion of atypical KD on the $9^{\text {th }}$ day of the illness because of persisting clinical conditions including fever and jaundice. Bilateral diffuse dilatation of the coronary arteries (CAs) (3.3 $\mathrm{mm}$ left and $3.6 \mathrm{~mm}$ right) and minimal mitral valve insufficiency were detected on echocardiography. Patient was treated with single dose of intravenous immunoglobulin (IVIG, $2 \mathrm{~g} / \mathrm{kg} /$ day) and acetylsalicylic acid (ASA, $80 \mathrm{mg} / \mathrm{kg} /$ day for the first 10 days). On the $11^{\text {th }}$ day of illness desquamation of fingers and bilateral conjunctival erythema 
had occurred. His fever resolved after IVIG treatment within 24 hours and bilirubin and GGT levels returned to normal levels. Repeated abdominal and chest ultrasonography revealed no pelvic or pleural fluid. After $8^{\text {th }}$ day of high dose ASA treatment control echocardiography demonstrated an increase of CAs diameters (left $5.6 \mathrm{~mm}$ and right $3.5 \mathrm{~mm}$ ). Low-molecularweight heparin (LMWH) was added to the treatment. He was discharged after 32 days of hospitalization with the antiaggregant dose of ASA (5 mg/kg/day) and LMWH. The dilated CAs gradually improved on repeated echocardiograhic investigations. A one year outpatient follow-up revealed no remarkable additional morbidity or recurrence of $\mathrm{KD}$.

\section{Case 2}

A previously healthy 2.5-year-old boy was admitted to hospital with seven days of fever, swelling and redness of upper surface of foot. He had $39{ }^{\circ} \mathrm{C}$ fever, tenderness and indurative edema of feet and icter on physical examination. The medical history of the patient was unremarkable. Laboratory findings were as follows; hemoglobin $10.1 \mathrm{~g} / \mathrm{dl}$, WBC 22,400/ $\mathrm{mm}^{3}$, platelet count $413,000 / \mathrm{mm}^{3}$, ESR 145 $\mathrm{mm} / \mathrm{h}$, CRP $360 \mathrm{mg} / \mathrm{dl}$, ALT $51 \mathrm{U} / \mathrm{L}$, AST 44 $\mathrm{U} / \mathrm{L}$, total bilirubin $5.4 \mathrm{mg} / \mathrm{dl}$, direct bilirubin $4.1 \mathrm{mg} / \mathrm{dl}$, GGT $444 \mathrm{U} / \mathrm{L}$. Urinalysis revealed the presence of pyuria. Cultures of blood, urine and throat were negative. Abdominal ultrasonography was normal. Serological tests for hepatitis A, B, C, EBV and cytomegalovirus were negative for acute infections. In light of the findings of fever for seven days and cholestasis without apperent causes, tenderness and indurative edema of feet, atypical KD was considered. The echocardiographic investigation that was performed on the second day of hospitalization demonstrated irregularity and dilatation of left CA ostia and mitral valve insufficiency. A single dose of IVIG (2 g/ $\mathrm{kg} /$ day $)$ and ASA $(80 \mathrm{mg} / \mathrm{kg} /$ day, for the first 10 days) were administered. Thereafter, bilateral conjunctival erythema developed. After 24 hours of treatment, clinical and laboratory improvement was rapidly obtained with regression of fever and bilirubin levels. ASA was reduced to an antiaggregant dose after 10 days. The patient was discharged with sequela of mild left CA dilatation and minimal mitral valve insufficiency. A two month follow- up revealed no recurrence of fever or additional increase of CA diameters with antiaggregant dose of ASA.

\section{Discussion}

Kawasaki disease is characterized by fever for at least five days and four or more of the following five major clinical features; bilateral nonexudative conjunctival injection, erythema of the lips and oral mucosa, changes in the extremities, polymorphous skin rash, and unilateral painless cervical lymphadenopathy. ${ }^{1,2}$ The patients who do not fulfill the major diagnostic criteria yet having echocardiographic findings have been defined as incomplete $\mathrm{KD}$ and who present with atypical conditions have been defined as atypical KD. ${ }^{1}$ Although our first case had none of these classical criteria, febrile cholestasis without an apparent cause was given rise to the diagnosis of $\mathrm{KD}$. The second case had one of the classical criteria additionally.

Although gastrointestinal involvement does not belong to the classic diagnostic criteria; diarrhea, various degrees of abdominal pain, hepatic dysfunction, hydrops of gallbladder up to $15 \%$ of patients and acute febrile cholestatic jaundice are reported in patients with KD. 1,3 The presentation with acute febrile cholestasis are among the atypical KD. Hydrops of the gallbladder as an acute acalculous, noninflammatory condition can occur in infants and children. On the other hand, acute acalculous cholecystitis is uncommon in children and is usually caused by infection especially streptococci, Staphylococcus aureus, Salmonella spp, Leptospira interrogans, EBV, hepatitis A virus, Ascaris or Giardia lamblia. 4,5 Both of our patients had biochemical findings of acute cholestasis without the evidence of an enlarged, thick-walled gallbladder, without calculi on ultrasonographic investigation. Hepatotropic viral infections and drugs were also excluded. It was postulated that a vasculitis of $\mathrm{KD}$ by the inflammatory changes of liver and gallbladder, and obstructive mechanism with enlarged lymph nodes gave cause to cholestasis. ${ }^{6}$

In a study that reviewed acute febrile cholestatic jaundice, KD was found as the second most frequent (20\%) cause among the 24 children. All of these patients had 
presented with hepatomegaly, elevated acute phase reactants, aminotransferase levels and cholestatic markers. ${ }^{7}$ In an another study from Spain three of the 31 cases with KD had jaundice, higher levels of ALT, AST and GGT without abnormalities in liver, gallbladder and intrahepatic or extrahepatic bile ducts as shown by ultrasound. ${ }^{8}$ In a recent study from Turkey, 35 patients with KD were described. None of these patients had acute cholestasis as the initial feature of KD. ${ }^{9}$

It is well known that $\mathrm{KD}$ is a leading cause of acquired heart disease in children CA aneurysms occurring in $20 \%$ to $25 \%$ of children who do not receive IVIG treatment. In children who present with incomplete $\mathrm{KD}$, the risk of CA sequelae is higher than classical KD. Records of patients with KD and CA aneurysms were reviewed at four United States centers from 1981 to 2006. Of 195 patients, 137 (70\%) had had classical KD, and 53 (27\%) had had incomplete KD. Authors investigated the performance of the 2004 American Heart Association (AHA) statement recommendations in this retrospective cohort including 53 eligible patients with incomplete KD for IVIG treatment. They concluded that application of the 2004 AHA recommendations, compared with the classic criteria alone, improves the rate of IVIG treatment for patients with KD who develop CA aneurysms. ${ }^{10}$ Standard treatment in the acute stage of $\mathrm{KD}$ includes combination of single high dose $(2 \mathrm{~g} / \mathrm{kg})$ IVIG and high-dose aspirin. The patients who respond to initial treatment of IVIG, fever resolves within 24 to 48 hours. However, approximately $10 \%$ of the patients have persistent or recurrent fever beyond 36 hours of therapy and require further treatment.1,2 In our two patients with incomplete KD and CA aneurysm had responded to initial IVIG treatment and further treatment was not required. Acute management of patients with CA aneurysm should be based on the extent and severity of the lesion. Although low dose ASA is sufficient for mild disease, because of the increased risk of thrombosis through CA, additional therapy such as LMWH, heparin or antiplatelet agent may be required for severe disease management. ${ }^{2}$ In our first case, because of the presence of a large aneurysm, combination of ASA and LMWH were required for long-term treatment.
In conclusion: atypical presentations of $\mathrm{KD}$ like acute febrile cholestatic disease should be kept in mind for timely diagnosis and treatment of children with incomplete KD. Early IVIG administration for a febrile syndrome of unknown etiology in a child when jaundice is present, should be considered. The antiaggregant and anticoagulant therapies are useful in the long-term management of $\mathrm{KD}$ patients with CA dilatation.

\section{REFERENCES}

1. Newburger JW, Takahashi M, Gerber MA, et al. Diagnosis, treatment, and long-term management of Kawasaki disease: a statement for health professionals from the Committee on Rheumatic Fever, Endocarditis, and Kawasaki Disease, Council on Cardiovascular Disease in the Young, American Heart Association. Pediatrics 2004; 114: 1708-1733.

2. Freeman AF, Shulman ST. Kawasaki disease: summary of the American Heart Association guidelines. Am Fam Physician 2006; 74: 1141-1148.

3. Zulian F, Falcini F, Zancan L, et al. Acute surgical abdomen as presenting manifestation of Kawasaki disease. J Pediatr 2003; 142: 731-735.

4. Suchy JF. Diseases of the Gallbladder. In: Kliegman RM, Stanton BF, St Geme JW, Schor NF (eds). Nelson Texbook of Pediatrics (20 th ed). Philadelphia: Saunders, 2015: 1971-1972.

5. Alkhoury F, Diaz D, Hidalgo J. Acute acalculous cholecystitis (AAC) in the pediatric population associated with Epstein-Barr Virus (EBV) infection. Case report and review of the literature. Int J Surg Case Rep 2015; 11: 50-52.

6. Valentini P, Ausili E, Schiavino A, et al. Acute cholestasis: atypical onset of Kawasaki disease. Dig Liver Dis 2008; 40: 582-584.

7. Taddio A, Pellegrin MC, Centenari C, Filippeschi IP, Ventura A, Maggiore G. Acute febrile cholestatic jaundice in children: keep in mind Kawasaki disease. J Pediatr Gastroenterol Nutr 2012; 55: 380-383.

8. Ibáñez-Alcalde M, Sánchez-Forte M, Giménez-Sánchez F, Ortega-Montes Á, Martínez-Espinosa G. Cholestasis as the initial feature of Kawasaki disease. Pediatr Infect Dis J 2012; 31: 766-767.

9. Aldemir-Kocabaş B, Karbuz A, Karadeniz C, et al. Another face of Kawasaki disease. Turk J Pediatr 2014; 56: 392-398.

10. Yellen ES, Gauvreau K, Takahashi M, et al. Performance of 2004 American Heart Association recommendations for treatment of Kawasaki disease. Pediatrics 2010;125: e234-41. 